\title{
A STUDY ON ARTIFICIAL INTELLIGENCE IN THE TIMES OF COVID-19
}

\author{
Boosarapu Asmika, Assistant professor of CSE Department, GITAM University, Hyderabad,
}

\section{Telangana \& India. asmika.ashu@gmail.com}

Abstract- the COVID-19 caused by SARS-CoV-2 (severe acute respiratory syndrome coronavirus 2) has already taken pandemic portion of the world by spreading more than 100 countries considerably within few weeks. And created devastation on the human civilization. So, the precise and expeditious diagnosis of COVID-19 cases plays a crucial role in timely quarantine and Healthcare, which requires the support of decisive technology like Artificial Intelligence. In this paper I am presenting a study on AI based methodologies that are being widely used in various applications in the battle against the deadly coronavirus outbreak and summarises the crucial roles of AI in this unusual fight. This outcome driven technology is applied in number of areas where AI plays an essential role from screening, analysing and diagnosing the risk to detect the cluster of cases. It can also be used to predict the cases in future and also be applied to keep track of number of current cases, recovered cases and death cases. The technologies based on artificial intelligence are applied for the delivery of exceptional services like discovery of medicines for cure, replacing man power at hospitals by AI based robots for serving medicines and food, disinfecting surfaces, in tackling the Covid-19 outbreak.

Keywords - AI, Artificial Intelligence, Coronavirus, COVID-19, Diagnosis, Outbreak, Pandemic, SARS-CoV-2.

\section{INTRODUCTION}

The first human case of novel coronavirus was emerged in Wuhan city, China, which causes acute respiratory illness and even fatal acute respiratory distress syndrome (ARDS). The new coronavirus named as SARS-Cov-2 by International Committee on Taxonomy of Viruses (ICTV) [1] and the infectious diseases infected by this coronavirus was named as Coronavirus Disease 2019 (COVID-19) by World Health Organization (WHO) [2]. The update till June 21, 2020, 12:54pm CEST the worldwide Coronavirus cases are 8, 708,008, Death cases are 461,715 [3].

This virus has made a severe impact in the health sector globally. All over the world, most of the countries have initiated different policies to ease the impact of the COVID19 pandemic.

Artificial Intelligence is such a technology. Which can be used to fight in battle against deadly COVID-19 outbreak. AI is being applied and delivering results in Detecting, Predicting the virus and number of people affected, Monitoring positive cases and survivals, precautions to be taken for Preventing, deploying robots to reduce further level transmission, Drug and Vaccine development to the present pandemic COVID-19. This AI driven solutions to COVID-19 includes the management of services and resources at healthcare centers. AI framework solutions incorporate screening, analysing and diagnosing the risk factor of a patient, prevent community transmission by employing robots at hospitals for different purposes like delivering treatment and monitoring patient, detect the cluster transmissions and avoiding them for further spread, predict the future cases and keeping the track of current, recovered and death cases. The below figurel shows the role of Artificial intelligence to help combat covid-19. It demonstrates the complete role of AI in understanding the virus and accelerating medical research on drugs and treatments detecting and diagnosing the virus, and predicting its evolution assisting in recovery and preventing or slowing the virus spread through continuous monitoring and surveillance, and identifying contaminant regions, responding to health emergency by using individual information and monitoring the recovery which may probably improve the early signs of detection.

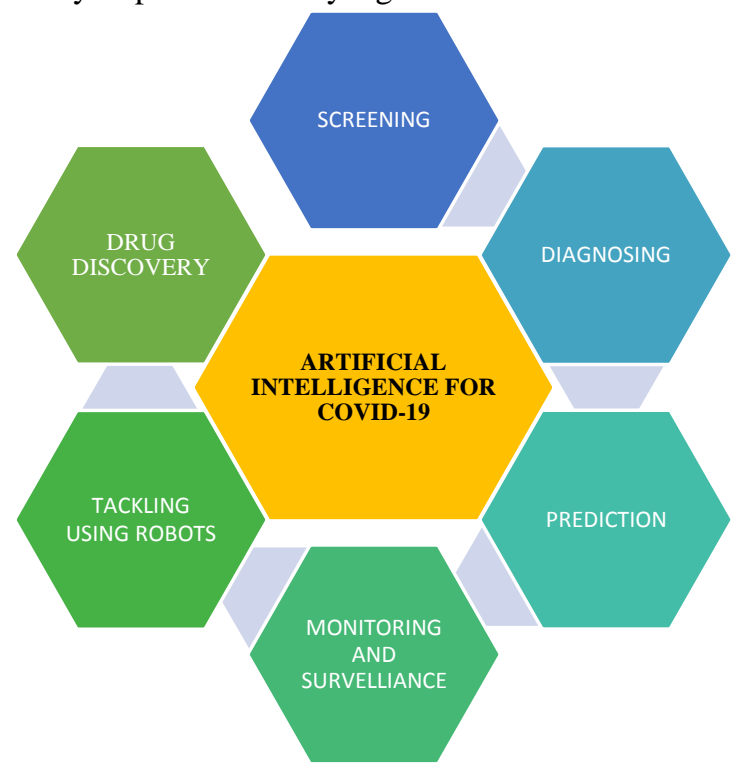

Figure 1: Role of Artificial intelligence in tackling covid-19 


\section{A STUDY ON AI METHODS AND APPLICATIONS}

\section{SCREENING USING AI FRAMEWORK}

COVID-19 can be diagnosed using built-in smartphone sensors. It is a simple and low-cost solution, since most of radiologists are using different gadgets for different dailypurposes. Today with the advent of new technologies we can enhance the user interface and user experience using frontend frameworks, even an ordinary person can use their smartphones for the screening of early symptoms of virus detections. The mobile devices we use today are exceptionally powerful which uses advanced processors added with neural processor units which is a combination of CPU and GPU (control processing unit and graphics processing unit) which is an AI accelerator, embedded in the devices and used for screening of multiple diseases depending upon characteristics, processing and added with number of sensors including cameras, microphone, color sensor, fingerprint sensor, humidity-sensor, wireless chipsets and proximity sensors etc, each of which has a functionality in collecting and detecting various symptoms for example camera and inertial sensors used for anlysing images and neck postures to predict the headache level [4], microphone is used to detect type of cough [5] and fingerprint sensor can be used to detect body temperature. The Artificial Intelligence (AI) qualified framework reads the smartphone sensors signal measurements to predict the category of severity because it may vary from person to person and predict the best possible outcome of the disease.

\section{DIAGNOSING COVID-19 USING AI}

The medical diagnosis of novel coronavirus is mainly depends on transmission and control history of disease, manifestation of disease and some additional respiratory symptoms like cough, fever, breathlessness and viral pneumonia. Therefore, auxiliary examinations are necessary for the diagnosis of COVID-19, just as the epidemiological history [6], [7]. Chest CT (Computed Tomography) has a high susceptibility for diagnosis of COVID-19. In the current scenario the primary tool for the detection of COVID-19 is Chest CT [8], [9], [10].

Feng Shi et al, [11] proposed the AI-based image acquisition can significantly help automate the scanning process and also reshape the workflow with minimal contact to patients, providing the simplest protection to the imaging technicians. Also, Artificial Intelligence can improve effectiveness of work by accurate depiction of infections in X-ray and CT images. Furthermore,the computer-aided platforms helps radiologists in making medical decisions such as disease diagnosis, prediction and tracking. To delineate the latest progress of medical imaging and radiology fight against COVID-19, the integrated approach of AI with X-ray and CT are tremendously used in the frontline hospitals .

\section{AI BASED ROBOTS IN FIGHT AGAINST COVID-19}

Guang-Zhong Yang et al.[12] described the role of Robots in fight against covid-19 which is shown in figure2. To prevent community level transmission of COVID-19, physical distancing is recommended but which has become a biggest challenge at hospitals. Most of the works at hospitals are taken care manualy like delivering treatment to infected people, suppling food and medicines, disinfecting surfaces, this may put work force at high exposure to infection. So an alternate and best solution is deploying Robots, and they play a vital role and are capable enough to replace humans. Robots can be used for delivering medications and food, disinfection, measuring vital signs. The robot-controlled noncontact ultraviolet (UV) surface disinfection has been used for disease prevention because COVID-19 spreads not only from Droplet transmission, which occurs when a person is in close or direct contact with person who has been suffered with respiratory problems such as coughing or sneezing and is consequently at risk of having his/her mucosae (mouth and nose) or conjunctiva (eyes) exposed to potentially infective respiratory droplets. One of the reasons for transmission is immediate environment around the infected person. Therefore, the community level transmission of coronavirus is not the only possibility for the spread of the pandemic. The another reason for the spread is unintended and partial contact with surfaces in the immediate environment or with objects used on the infected person for example stethoscope or thermometer [13]. Coronaviruses can exist on inanimate surfaces - including plastic, glass and metal-for days, and UV light devices such as pulsed xenon ultraviolet have been proved to be effecient in diminishing contamination on frequently contacted surfaces at hospitals. Inorder to reduce the human exposure to the pandemic especially in the case of manual disinfection, which requires labour force mobilization and increases risk to overcome this, cleaning personnel, independent or remote-controlled disinfection robots could lead to effective, fast and cost-effective disinfection [14]. For screening and diagnosis at public areas mobile robots can be used to screen multiple people concurrently. These mobile robots could also be used to monitor temperatures of in or/and out patients frequently in various areas of the health centres and hospitals with data linked to hospital database

\section{MONITORING AND SURVEILLANCE}

AI can help calculating the probability of infection spread by this virus identifying the cluster of cases. It can predict future course of disease and likely strike back again by analyzing past and present medical, molecular and scientific data. Furthermore, AI can be applied to predict the number of positive cases and deaths in a particular 
region and it can forecast contamination in various regions by using this processed collection of data.
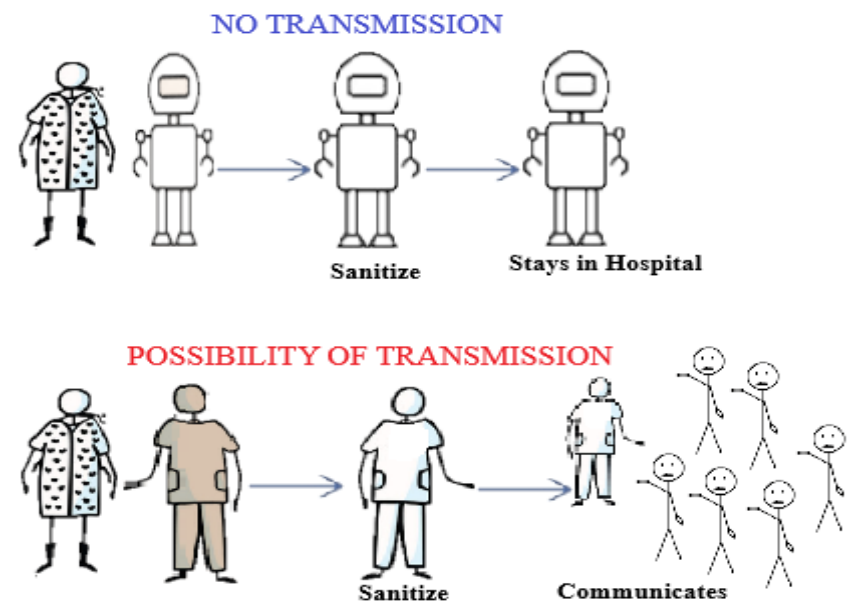

Figure 2: How robots prevent community level transmission

\section{AI IN DRUG DISCOVERY}

The Applications of Artificial Intelligence has been extended to Drug discovery. AI has a vital role in fight against COVID-19 from diagnosis to finding all possible ways to tackle pandemic disease using non-interfering devices, and generate predictions on patient results based on in and out data records including electronic health records. AI has the potential to solve medical challenges created by the COVID-19 pandemic that is being shown in the below figure, it identifying required molecules from data, find drug target, and can also be used to predict the structure of coronavirus related proteins, can find existing drugs that may be reconsidered to treat this pandemic COVID-19, and to develop new compound that may be favourable for drug development [15]. The below figure 3 explains $\mathrm{AI}$ in the process of drug discovery.

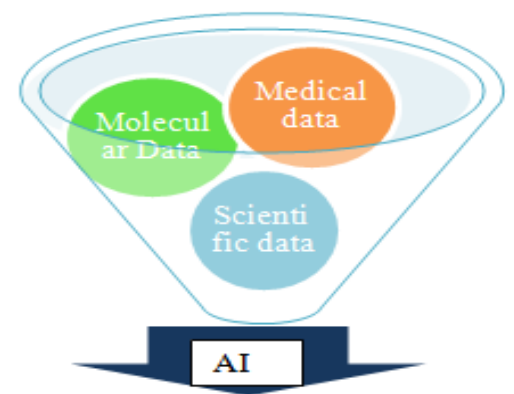

Good Molecules, Drug target, COVID-19 related Proteins
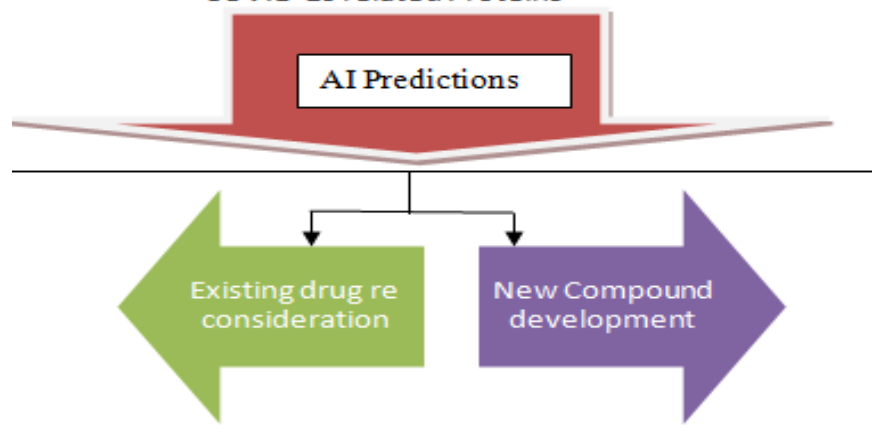

Figure 3: AI in the process of drug discovery

\section{PERFORMANCE ANALYSIS}

\section{HOW AI IMPROVED SCREENING AND DIAGNOSING OF COVID-19?}

Screening of COVID-19 usually done manually, which is time consuming and the challenge is there are not enough medicos and kits for Screening of COVID-19 pandemic as it is highly contagious and it is impossible to reach and check each and every individual for infection. By using our AI powered smart phones one can self-check for particular symptoms. If the maximum number of symptoms matches then there are high chances of infection and needs to be self-isolated and contact the nearest health centre for further analysis and treated accordingly. Hence it increases the performance by reducing involvement of medical staff and accurate prediction for future diagnosing.

The traditional diagnosis methods such as reverse transcriptase polymerase chain reaction (RT-PCR) has failed to diagnose COVID-19 accurately and facing challenges as the number of cases increasing day by day. Chest computed tomography is best way to diagnose patients of novel coronavirus. CT alone may have limited negative predictive value for ruling out SARS-CoV-2 infection, as some patients may have normal radiological findings at early stages of the disease [16]. AI has improved diagnosing process by collecting and analysing data of CT and is effectively used for rapid diagnosing across most affected countries like Russia, USA, China and European countries. Hence, diagnosis of pandemic covid-19 is accurately detected by AI in collaboration with clinical methods such as CT.

\section{HUMANS VERSUS ROBOTS IN SURVEILLANCE AND MONITORING WITH AI}

Humans have a tendency to bear optimal workload for best results, whereas increasing number of cases involves huge work force and workload for treating COVID-19. Here comes AI enabled robots with better performance by working continuously and effectively. It also reduces human work load, and also breaks chain of human to human transmission.

By deploying thermal sensing cameras at public places like airports, railway stations, bus depots, shopping malls, hospitals etc., AI has detected successfully the level of contamination by surveying data of past travel and medical history and the contacts of the infected humans. Monitoring of patients infected by COVID-19 with the help of automatic surveillance robots at hospitals within specified time intervals can make it easier for diagnosing, delivering food and sanitizing surfaces. These measures implemented during the pandemic has proven efficiency in containing virus. 


\section{CONCLUSION}

Artificial Intelligence is playing a vital role in several stages of novel coronavirus, every role is important in battle against this pandemic based on stage where we are in actual cycle of pandemic from predicting spread of COVID-19 to replacing man power at hospitals with robots. Countries with higher population like China, India, Russia, USA, Brazil, European nations etc., has a surge in number of cases increasing daily. Until the discovery of drug for the treatment of COVID-19, we are bound to use AI technology in possible ways for rapid testing, implementing infrastructure for treatment. The more possibility of reducing human contact the more prevention of spread is possible in present situation. This paper reviews how the AI Driven Technology that is being used for self-screening at homes using smart phones by using advanced frame works, rapid diagnosis using AI enabled CT scan for accurate results, detecting symptoms like temperature at public places using thermal sensing devices, how robots are used at hospitals for treatment, delivering food and sanitizing surfaces and artificial intelligence is being used as a potential source for drug discovery with the help humongous amount of medical, molecular, public and private data collected. Further this technology can be enhanced not only in detecting COVID-19 but also other communicable viral diseases. This technology has the potential to tackle without the intervention of humans and likewise diseases can also be contained in future.

\section{REFERENCES}

[1] International committee on taxonomy of viruses

[2] World health organization (WHO) website, "Naming the coronavirus disease (COVID-19) and the virus that causes it".

[3] World health organization coronavirus disease (COVID-19) dashboard.

[4] Worawat Lawanont, Masahiro Inoue, P. Mongkolnam, Chakarida Nukoolkit, "Neck posture monitoring system based on image detection and smartphone sensors using the prolonged usage classification concept" IEEJ Transactions on Electrical and Electronic Engineering, 13(10), 1501-1510, DOI:10.1002/TEE.22778, 2018.

[5] Sudip Vhaduri,Theodore Van Kessel, Bongjun Ko, David Wood, "Nocturnal Cough and Snore Detection in Noisy Environments Using Smartphone-Microphones", IEEE International Conference on Healthcare Informatics (ICHI), June 2019, DOI: 10.1109/ICHI.2019.8904563.

[6] Xiaowei Li, Manman Geng, Yizhao Peng, Liesu Meng,Shemin Lu, "Molecular immune pathogenesis and diagnosis of COVID-19", Journal of Pharmaceutical Analysis Volume 10, Issue 2, April 2020.

[7] F Soares, A Villavicencio, MJ Anzanello, FS Fogliatto, "A novel high specificity COVID-19 screening method based on simple blood exams and artificial intelligence", medRxiv2020, doi:https://doi.org/ 10.1101/2020.04 .10.20061036.

[8] Abdul Waheed, Muskan Goyal, Deepak Gupta, Ashish Khanna, Fadi Al-Turjman, Plácido Rogerio Pinheiro , "CovidGAN: Data Augmentation Using Auxiliary Classifier GAN for Improved Covid-19 Detection ”, IEEE vol 891916
91923 , 14 May 2020, DOI:10.1109/ACCESS.2020.299476 2

[9] Tao Ai, Zhenlu Yang, HongyanHou, Chenao Zhan, Chong Chen, WenzhiLv, Qian Tao, Ziyong Sun, Liming Xia, "Correlation of Chest CT and RT-PCR Testing in Coronavirus Disease 2019 (COVID-19) in China: A Report of 1014 Cases", Feb 26 2020, doi:https://doi.org/10.1148/radiol. .2020200642 .

[10] Geoffrey D. Rubin , Christopher J. Ryerson, Linda B. Haramati, Nicola Sverzellati, Jeffrey P. Kanne, SuhailRaoof, Neil W. Schluger, Annalisa Volpi, Jae-JoonYim, Ian B. K. Martin, Deverick J. Anderson, Christina Kong, TalissaAltes, Andrew Bush, Sujal R. Desai, Jonathan Goldin, Jin Mo Goo, Marc Humbert, Yoshikazu Inoue, Hans-Ulrich Kauczor, Fengming Luo, Peter J. Mazzone, Mathias Prokop, Martine Remy-Jardin, Luca Richeldi, Cornelia M. Schaefer-Prokop, Noriyuki Tomiyama, Athol U. Wells, Ann N. Leung, "The Role of Chest Imaging in Patient Management during the COVID-19 Pandemic: A Multinational Consensus Statement From the Fleischer Society", Apr 7 2020, doi:https://doi.org/ 10. 1148/radiol .2020201365.

[11] Feng Shi, Jun Wang, Jun Shi, Ziyan Wu, Qian Wang, Zhenyu Tang, KeleiHe, Yinghuan Shi, Dinggang Shen, "Review of Artificial Intelligence Techniques in Imaging Data Acquisition, Segmentation and Diagnosis for COVID-19", IEEE Reviews in Biomedical Engineering (2020),16 April 2020. DOI:10.1109/RBME.2020.2987975.

[12] Guang-Zhong, Yang,Bradley, J. Nelson, Robin R. Murphy, Howie Choset, Henrik Christensen, steven $\mathrm{H}$. Collins, Paolo Dario, Ken Goldberg, Koji Ikuta, Neil Jacobstein, Danica Kragic, Russell H. Taylor and Marcia McNutt, "Combating COVID-19-The role of robotics in managing public health and infectious diseases", Science Robotics 25 Mar 2020,Vol. 5, Issue 40, eabb5589. DOI: 10.1126/scirobotics.abb5589

[13] World Health Organization "Modes of transmission of virus causing COVID-19".

[14] C. R. Kovach, Y. Taneli, T. Neiman, E. M. Dyer, A. J. A. Arzaga, S. T. Kelber, "Evaluation of an ultraviolet room disinfection protocol to decrease nursing home microbial burden, infection and hospitalization rates. BMC Infect. Dis. 17, 186 (2017). DOI:https://doi.org/10.1186/s12 879-0172275-2.

[15] Joseph Bullock, Alexandra Luccioni, Katherine Hoffmann Pham, Cynthia Sin Nga Lam, Miguel Luengo-Oroz, "Mapping the Landscape of Artificial Intelligence Applications against COVID-19”. ArXiv: 2003.11336.

[16] Xueyan Mei, Hao-Chih Lee, Kai-yue Diao, Mingqian Huang, Bin Lin, Chenyu Liu, Zongyu Xie, Yixuan Ma, Philip M. Robson, Michael Chung, Adam Bernheim, Venkatesh Mani, Claudia Calcagno, Kunwei Li, Shaolin Li, Hong Shan, Jian Lv, Tongtong Zhao, Junli Xia, Qihua Long, Sharon Steinberger, Adam Jacobi, Timothy Deyer, Marta Luksza, Fang Liu, Brent P. Little, Zahi A. Fayad \& Yang Yang, "Artificial intelligence-enabled rapid diagnosis of patients with COVID-19", Nat Med (2020), DOI:https://doi.org/10.1038/s41591-020-0931-3. 